\title{
The Mechanism Study of Dracocephalum Moldavica L. Total Flavonoids on Apoptosis induced by Myocardial Ischemia/Reperfusion injury in vivo and in vitro
}

\author{
Wenjiang Cao ${ }^{1 \#}$, Yong Yuan ${ }^{1 \#}$, Yangyang Wang², Li Tian², Xinchun Wang1,2 ${ }^{*}$, Jianguo Xin ${ }^{3 *}$, Yanfang \\ Wang ${ }^{2}$, Xinhong Guo ${ }^{1}$ and Dongmei Qin ${ }^{2}$ \\ ${ }^{1}$ First Affiliated Hospital of the Medical College, China \\ ${ }^{2}$ Pharmacy of College, China \\ ${ }^{3}$ Xinjiang Institute of Materia Medica, China \\ \#These authors contributed equally to this work \\ *Corresponding author's: Xinchun Wang, First Affiliated Hospital of the Medical College, Pharmacy of College, China \\ Jianguo Xing, Xinjiang Institute of Materia Medica, China
}

\section{ARTICLE INFO}

Received: 㠅 July 19, 2019

Published: 慧 August 07, 2019

Citation: Wenjiang Cao, Yong Yuan, Yangyang Wang, Li Tian, Xinchun Wang, Jianguo Xin, Yanfang Wang, Xinhong Guo, Dongmei Qin. The Mechanism Study of Dracocephalum Moldavica L. Total Flavonoids on Apoptosis induced by Myocardial Ischemia/Reperfusion injury in vivo and in vitro. Biomed J Sci \& Tech Res 20(3)-2019. BJSTR. MS.ID.003443.

Keywords: Dracocephalum moldavica L.; myocardial ischemia/reperfusion injury; apoptosis; PI3K/Akt; Erk1/2; P38 MAPK

\begin{abstract}
Dracocephalum moldavica $L$. is a commonly medicinal plant of Uygur and Mongolian nationalities and has a medicinal history for hundreds of years. It has been confirmed to have protective effects against myocardial ischemia-reperfusion injury, but its mechanism is still unknown. To investigate the protective effect of total flavonoids extracted from Dracocephalum moldavica L. (TFDM) on apoptosis in myocardial ischemia/reperfusion injury (MIRI) and reveal its potential mechanism in vivo and vitro. The MIRI model was established by 30 min ischemia and 180 min reperfusion in SD rats. The hypoxia/ reoxygenation $(\mathrm{H} / \mathrm{R})$ model was established by $3 \mathrm{~h}$ hypoxia and $2 \mathrm{~h}$ reoxygenation using H9c2 cell in vitro. The TFDM was extracted and analyzed by HPLC. The infract size, CK-MB, LDH, AST, histopathological, apoptosis, apoptosis-related proteins, RISK and P38 MAPK pathway related proteins were measured.

The results showed that TFDM markedly reduced infract size, declined CK-MB, LDH and AST, increased the cell viability of H9c2 cardiomyocytes, improved the histopathological, remarkably inhibited the apoptosis, whereas the anti-apoptotic effect of TFDM was reversed by LY294002 and PD98059, however SB203580 further inhibited myocyte apoptosis. TFDM up-regulated the expressions of Akt, Erk1/2, Gsk-3 $\beta$, meanwhile, inhibited the activation of P38 MAPK and down-regulated the expressions of apoptosis related proteins. Results indicated that TFDM has definite protection for apoptosis in MIRI, its mechanism is related to inhibit the apoptosis through mediation of RISK / GSK3 $\beta$ and P38 MAPK signal pathway.
\end{abstract}

\section{Introduction}

Acute myocardial infarction (AMI) is the first manifestation of coronary heart disease, which is a critical threat to cause disability and death of human being [1,2]. Early reperfusion is the most effective measure to salvage the ischemic myocardium and avoid infarction. However, paradoxically, prolonged reperfusion itself may aggravate myocardial damage, which lead to severer functional disturbances such as arrhythmias, myocardial stunning, microvascular obstruction et al than that in period of ischemia [3]. It even induces death of cardiomyocytes which would cause lethal reperfusion injury [4]. Recent years, studies have shown extensive apoptotic cardiomyocytes death is a key reason that results in myocardial ischemia/reperfusion injury (MIRI), the regulation of apoptosis is taken for a valid therapeutic strategy for easing MIRI. Hence, it is crucial to explore novel agents and elucidate the potential mechanism of it. 
PI3K/Akt and Erk 1/2 are the two key components of reperfusion injury salvage kinase (RISK) pathway, the activation of which has been confirmed to play an important role in cardiac protection [5]. Recently study suggested that PI3K/Akt and Erk 1/2 are closely related to the modulation of mitochondrial function and cardiomyocytes apoptosis [6]. P38 as the key member of MAPKs family, is also involved in myocardial apoptosis. Gsk-3 $\beta$, as a core downstream kinase, is regulated by other kinases including Akt and Erk1/2. The activated Gsk-3 $\beta$ can affect the mitochondrial permeability and regulate the ratio of $\mathrm{Bcl}-2 / \mathrm{Bax}$, then prevent the release of apoptotic factor cytochrome $c$ into the cytoplasm and the activation of caspase-dominated apoptotic pathway, finally inhibit the apoptosis, which induces cardio-protective effects [5,7]. Dracocephalum moldavica $L$. is an annual herbaceous plant of the dracocephalum, which belongs to labiate family [8]. Its entire grass is used frequently as a traditional Uygur folk medicine in Xinjiang of China for the treatment of coronary heart disease, hypertension, neuralgia and migraine $[9,10]$.

The total flavonoids extracted from Dracocephalum moldavica $L$. (TFDM) were demonstrated to be the primary constituent through phytochemical analysis, which also indicated that it was composed of many ingredients, including tilianin, rosmarinic acid and luteolin, etc. [11]. TFDM possesses the versatile and potent biological activity. Study has been proved that it has protective effects against myocardial ischemia/reperfusion injury, but its mechanism is still unknown $[10,12,13]$. Therefore, this research was designed for the first time to investigate whether TFDM could reduce apoptosis induced by MIRI and find its potential targets based on the RISK pathway and P38 MAPK pathway in vivo and vitro. We are supposed to reveal the function of PI3K/Akt, Erk1/2 and p38 MAPK pathway in cardioprotective effect of TFDM and explore its possible mechanism at molecular level.

\section{Materials and Methods}

\section{Reagents}

The Dracocephalum moldavica L. was obtained from Xin Jiang Institute of Medicine (Xin Jiang, China). LY294002 (an inhibitor of PI3K/ Akt) and Anti-active + pro Caspase-3 antibody, phosphorAkt (Ser473) and Akt, phosphor-Gsk-3 $\beta$ (Ser9) and Gsk-3 $\beta$ were purchased from Abcam. PD98059 (an inhibitor of Erk1/2), SB203580 (an inhibitor of p38 MAPK), p44/42 MAPK (Erk1/2) and Phospho-p44/42 MAPK (Erk1/2) (Thr202/Tyr204) were purchased from Cell signaling. P38 and p-P38, Bax and Bcl-2 Rabbit Polyclonal antibody, GAPDH and cytochrome c Rabbit Polyclonal antibody were provided by Proteintech. 2,3,5-triphenyltetrazolium chloride (TTC) was from Solarbio. The TUNEL apoptosis assay kit was supplied by Roche Group (Basel, Switzerland).

\section{Preparation of TFDM}

The Dracocephalum moldavica L. was purchased from Xin Jiang Institute of Medicine (Xin Jiang, China) and stored in a desiccated condition. The plant material was identified and authenticated as Dracocephalum moldavica $L$. by Professor Jianguo Xing, a pharmacognosist, from Xinjiang Institute of Materia Medica (Xin Jiang, China). Moldavica raw materials (3.3 kg) were dried and crushed into coarse powder, which was extracted with 20 times amount of $40 \%(\mathrm{v} / \mathrm{v})$ ethanol under heating reflux for $3 \mathrm{~h}$. After filtration, ethanol of extracted solution was removed by decompressing distillation method and concentrated liquid $(0.125$ $\mathrm{g} / \mathrm{mL}$ ) was collected in the end. The flavonoids of concentrate were eluted with ethanol by HPD600 macroporous adsorption resin column at different flow rates. Finally, 70\% ethanol elution was concentrated into extractum after vacuum drying procedure. The yield was $2.5 \%$. The plant extracts were conducted on the phytochemical analysis according to standard procedures and then reported the result [14].

\section{HPLC Analysis of TFDM}

HPLC analysis was carried out as described previously [10]. A Waters HPLC system (Waters, USA) was used. All the analyses were conducted by a SunFire C $18(4.6 \times 250 \mathrm{~mm}, 5 \mathrm{um}$, Waters, Milford, USA) column. The mobile phase composed of $0.1 \%$ formic acid $(\mathrm{v} / \mathrm{v})$ and acetonitrile with a gradient elution: 0-5 min, 80\%A; 5-45 $\min , 80-70 \% \mathrm{~A} ; 45-70 \mathrm{~min}, 20 \% \mathrm{~A} ; 70-80 \mathrm{~min}, 0 \% \mathrm{~A}$. The flow rate of the mobile phase was $1.0 \mathrm{~mL} \mathrm{~min}-1$, the column temperature was $35^{\circ} \mathrm{C}$ and auto-sampler temperature was $10^{\circ} \mathrm{C}$.

\section{Cell Culture}

The rat cardiomyocyte-derived H9c2 cell line (Fuheng Biotechnology, Shanghai China) were cultured in high glucose Dulbecco's modified Eagle's medium (DMEM; gibco, Thermo fisher, Suzhou, China), 10\% (v/v) fetal bovine serum (FBS; Gibco, New Zealand, USA), $10000 \mathrm{U} / \mathrm{ml}$ penicillin and $10000 \mu \mathrm{g} / \mathrm{ml}$ streptomycin (HyClone). The cell was cultured in carbon dioxide incubator containing $5 \% \mathrm{CO} 2$ at $37^{\circ} \mathrm{C}$.

\section{Hypoxia/Reoxygenation (H/R) Model and Treatments}

The H/R injury model was established as previously [15]. H9c2 cell was cultured with glucose-free DMEM and maintained in a three-gas incubator $\left(94 \% \mathrm{~N}_{2}\right.$ and $\left.5 \% \mathrm{CO}_{2}\right)$ at $37^{\circ} \mathrm{C}$ for $3 \mathrm{~h}$. Subsequently, the cells were incubated in normal medium in an incubator $\left(95 \%\right.$ air and $\left.5 \% \mathrm{CO}_{2}\right)$ at $37^{\circ} \mathrm{C}$ for $2 \mathrm{~h}$. The cell was pretreated with TFDM $(25,50$ and $100 \mu \mathrm{g} / \mathrm{ml})$ for $24 \mathrm{~h}$ before the

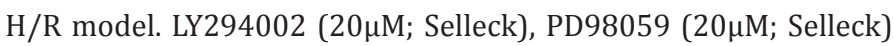
and SB203580 (10 $\mu \mathrm{M}$; Selleck) were added for $1 \mathrm{~h}$ before TFDM administration to intervene the effects of TFDM [16].

\section{Myocardial Ischemia/Reperfusion (I/R) Injury Model and Experimental Design}

The study used adult Sprague-Dawley rats (SPF, male, 230$280 \mathrm{~g}$ ) and the animals were administered adaptive feed for 7 days, then the MIRI model was performed as previously on the 8th day [17]. The experiment was divided into eight groups $(\mathrm{n}=$ 
80; 10 rats/group) as follows: Sham group, MIRI group, TFDM-L (15mg/kg) group, TFDM-M (30mg/kg) group, TFDM-H (60mg/kg) group, TFDM-H +LY2940029 (0.3g/kg) group, TFDM-H+PD98059 $(0.3 \mathrm{mg} / \mathrm{kg})$ group, TFDM-H+SB203580 (1.0mg/kg). The dosage of pharmacological blockers was decided according to previous study $[18,19]$. All rats were raised (4 per cage) at a condition of light/ dark cycle, under invariable temperature and humidity, and could get feed and water freely. All procedures were authorized by the Committee for the Ethical Use of Experimental Animals at Xin Jiang Medical University.

\section{Cell Viability Assay}

The cell viability of H9c2 cells was analyzed by a cholecystokinin-8 (CCK-8) assay. H9c2 cells were seeded on a 96-well plate and subjected with different treatments. Then $10 \mu \mathrm{l}$ of CCK-8 (Dojindo) was added and incubated the cell at $37^{\circ} \mathrm{C}$ for $2 \mathrm{~h}$. Finally, the absorbance was observed at the wavelength of $450 \mathrm{~nm}$ by a microplate reader (Varioskan Flash, Themo, USA).

\section{Assay of Myocardial Infarct Area}

At the end of experiments, we removed the heart rapidly. After washing out blood with normal saline, the left ventricle was separated and frozen at $-80^{\circ} \mathrm{C}$ for $10 \mathrm{~min}$, sliced it into $1.5 \mathrm{~mm}$ thick transverse sections, used $1 \%(\mathrm{w} / \mathrm{v})$ TTC (dissolved in $0.1 \mathrm{~mol} / \mathrm{L}$ phosphate buffer at $\mathrm{pH} 7.4$ ) to incubate them at $37^{\circ} \mathrm{C}$ for $20 \mathrm{~min}$ without light, and fixed them for $12 \mathrm{~h}$ in $10 \%(\mathrm{v} / \mathrm{v}$ ) formaldehyde to distinguish the viable myocardium from the necrotic myocardium. The viable tissue was stained red (area at risk, AAR), while the necrotic tissue was pale, unstained (infarct size area, IS) by TTC. We measured and imaged the area of infarct of each slice by digital imaging software (Image-Pro Plus Version 6.0). Percentage of infarct area was expressed as IS/AAR.

\section{Histological Examination of Myocardium}

After the reperfusion finished, rat tissues were harvest and fixed in $10 \%$ formalin solution. The heart tissue was processed into $5 \mathrm{~mm}$ slices. We used hematoxylin and eosin (H\&E) to stain the tissue and observed pathological changes of myocardium by microscopy at $200 \times$ magnification.

\section{The Content of Serum LDH, CK-MB and AST}

The blood was collected and centrifuged for serum. The LDH, CK-MB, AST assay kits (Jiancheng Bioengineering Institute, Nanjing, China) were taken to measure the level of them according to the manufacturer's instructions.

\section{Detection of Apoptosis with TUNEL}

The apoptosis was detected as previously [17]. Briefly, the tissues fixed in $4 \%$ formaldehyde for $48 \mathrm{~h}$, embedded in paraffin wax and cut into ultrathin section. The apoptosis was detected using TUNEL kit (Roche, Basel, Switzerland). The results were observed by microscopy (Olympus, Japan) at $400 \times$ magnification. The apoptotic index was expressed as the apoptotic myocytes number / the total myocytes number $\times 100 \%$.

\section{Flow Cytometry}

Cell apoptosis was quantified with the Annexin V-FITC Apoptosis Detection Kit (Multi Sciences Biotech, Hangzhou, China) according to the manufacturer's instructions. Briefly, the cell was washed twice using ice-cold PBS, resuspended in $500 \mu \mathrm{l}$ of Annexin $\mathrm{V}$ binding buffer, and stained with $5 \mu \mathrm{l}$ of Annexin V-FITC and $10 \mu \mathrm{l}$ of propidium iodide (PI) at room temperature for $5 \mathrm{~min}$ in the dark. Afterward, the fluorescent signals were measured using a flow cytometer (Becton-Dickinson, CA, USA).

\section{Western Blot}

Western blot analysis was finished as described early [17]. Briefly, the proteins were separated on a $10 \%$ SDS-PAGE $(100 \mu \mathrm{g} /$ lane) and then were transferred to PVDF membranes (Millipore, USA). Membranes were blocked with TBST or BSA at $25^{\circ} \mathrm{C}$ for $1 \mathrm{~h}$, incubated overnight at $4^{\circ} \mathrm{C}$ with the primary antibodies and then with HRP-conjugated secondary antibody at $25^{\circ} \mathrm{C}$ for $1 \mathrm{~h}$. Finally, the bands were analyzed by the Image J software (National Institutes of Health, MD, USA). GADPH was taken as internal reference.

\section{Statistical Analysis}

Statistical analysis was performed with SPASS 20.0 software (Chicago, IL, USA) and Origin pro 8 (OriginLab, Massachusetts, USA) software. The data were presented as means \pm standard deviation (SD), results were analyzed with one-way ANOVA. Statistical significance was considered when $\mathrm{P}<0.05$.

\section{Results}

\section{HPLC Profile of TFDM}

As shown in the Figure 1, the main components of TFDM were analyzed by HPLC, in comparison to standard sample, a kind of compound from TFDM was determined and analyzed, which was named tilianin and its content was quantified by HPLC. 

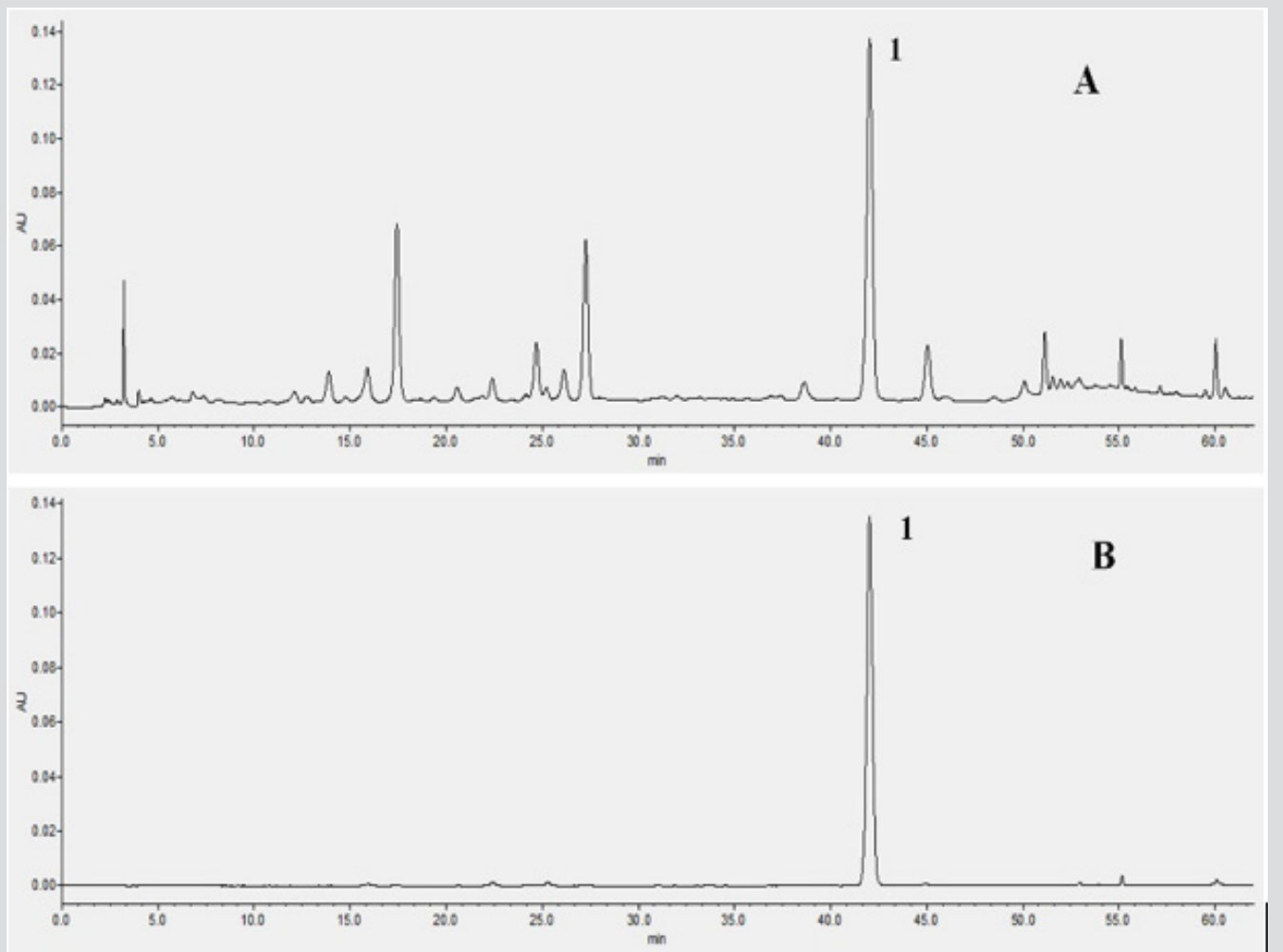

Figure 1: The HPLC analysis of TFDM. (A) The main components of TFDM. (B) the main components Tilianin in TFDM. 1 represent the Tilianin.

\section{TFDM Increased the Cell viability}

As shown in Figure 2, TFDM increased the viability of H9c2 cardiomyocytes with hypoxia/reoxygenation injury, the effects increased to a concentration-dependent manner, until to $100 \mu \mathrm{g} /$ $\mathrm{ml}$, and the $25,50,100 \mu \mathrm{g} / \mathrm{ml}$ were chosen as the low, medium and high concentration of TFDM.

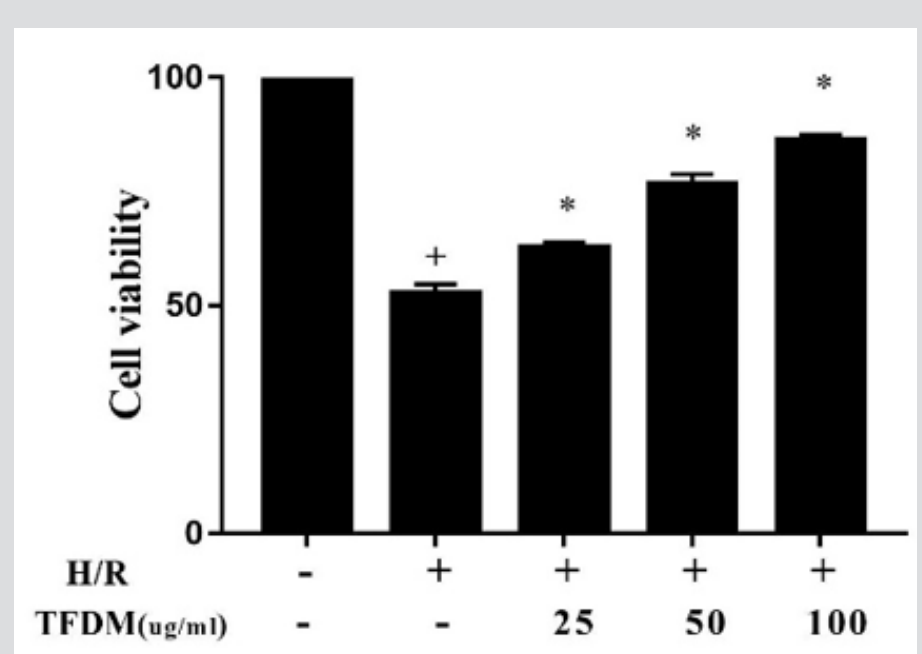

Figure 2: Effects of TFDM on H9c2 cell viability. Compared with the Norm group, $+\mathrm{P}<0.05$; Compared with the $\mathrm{H} / \mathrm{R}$ group,

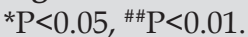

TFDM Reduced the Myocardial Infarction Area Induced by $\mathbf{I} / \mathbf{R}$

The results showed that there was a significant infarct induced by I/R injury in I/R group (Figure 3). In contrast, infarct size of
TFDM pretreatment group was reduced with improvement of administration dosage. Though the low dosage of TFDM did not display obvious decrease of infarct size compared with I/R group, the medium and high dose group remarkably reduced infarction area to $33.61 \pm 3.03 \%, 20.72 \pm 2.84 \%$ respectively. 


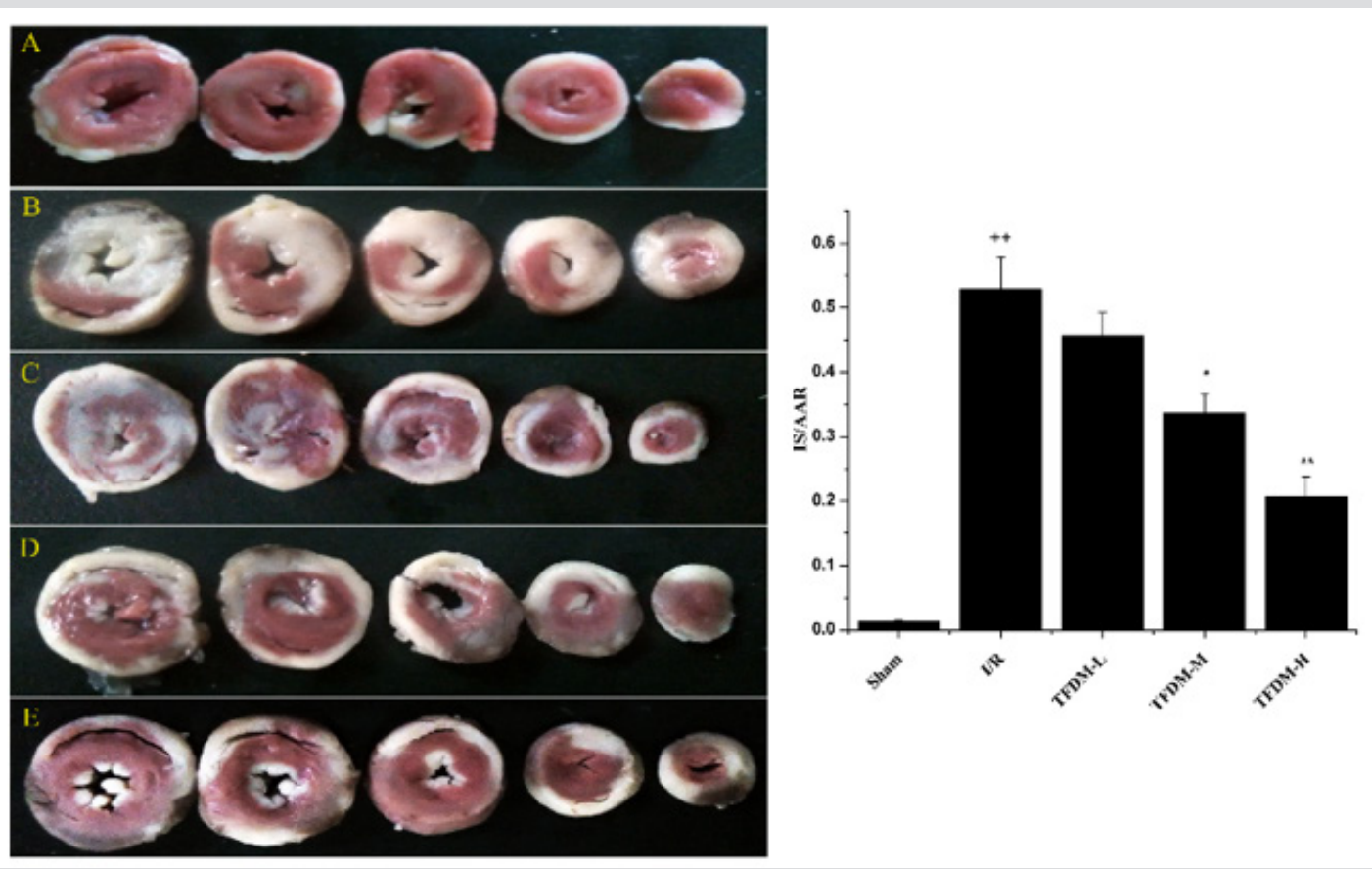

Figure 3: TFDM reduced the myocardial infarction area induced by I/R. A represented Sham groups. B represented I/R group. C represented TFDM-L group. D represented TFDM-M group. E represented TFDM-H group. Compared with the Sham group, $++\mathrm{P}<0.01$; Compared with the I/R group, ${ }^{*} \mathrm{P}<0.05, * * \mathrm{P}<0.01$.

\section{Effects of TFDM on Myocardial Histology}

As shown in Figure 4, conventional HE is staining was observed under light microscope, the cardiac muscle cells were arranged neatly and showed a bundle distribution, muscle fibers exhibited normal nuclear morphology and no cell swelling, necrosis. By contrast, myocardial tissue from I/R group displayed the extensive fusion lesions and neutrophil infiltration, muscle fibers showed fracture and necrosis, muscle membrane swelling and damaged. Compared with I/R group, pretreatment with TFDM could gradually reverse severe pathological changes induced by I/R injury. There was no significant difference between the group with low dosage of TFDM and I/R. After administration with the medium and high of TFDM, there was slight swelling and little structure turbulence.

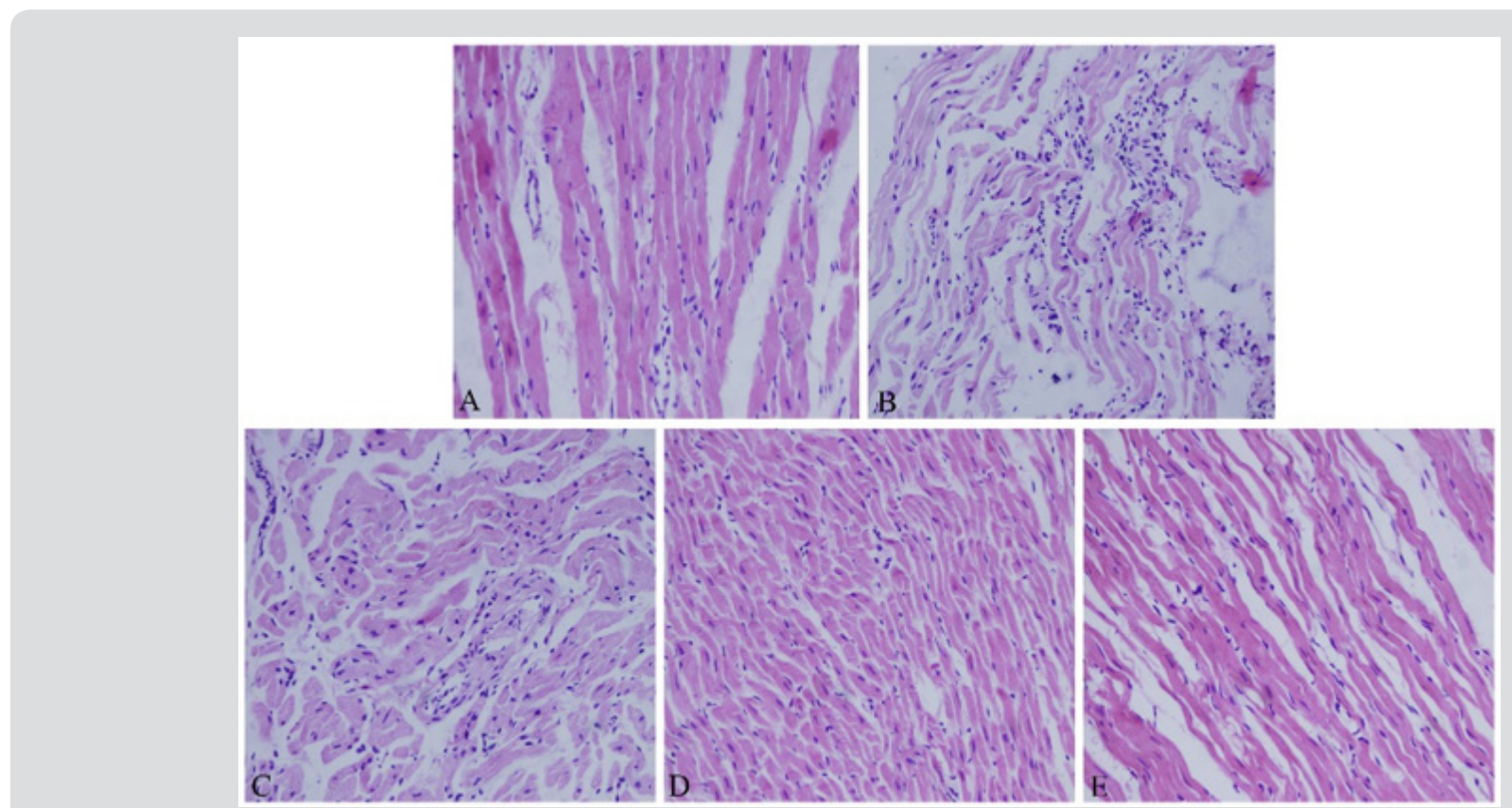

Figure 4: Effects of TFDM on myocardial histology. A represented Sham groups. B represented I/R group. C represented TFDM-L group. D represented TFDM-M group. E represented TFDM-H group. 


\section{Effects of TFDM on CK-MB, LDH and AST in Serum}

The content of myocardial enzyme directly reflects the degree of myocardial injury after I/R, the contents of serum CK-MB, LDH and AST were measured after 180 min of reperfusion. Results exhibited that a dramatic leakage of myocardial enzymes in the MIRI rats versus to Sham group. Compared with MIRI group, both the medium and high dosage of TFDM significantly reduced CK-MB, LDH and AST levels, however, the rats pretreated with low dosage of TFDM showed no significant change in comparison to I/R group (Figure 5).

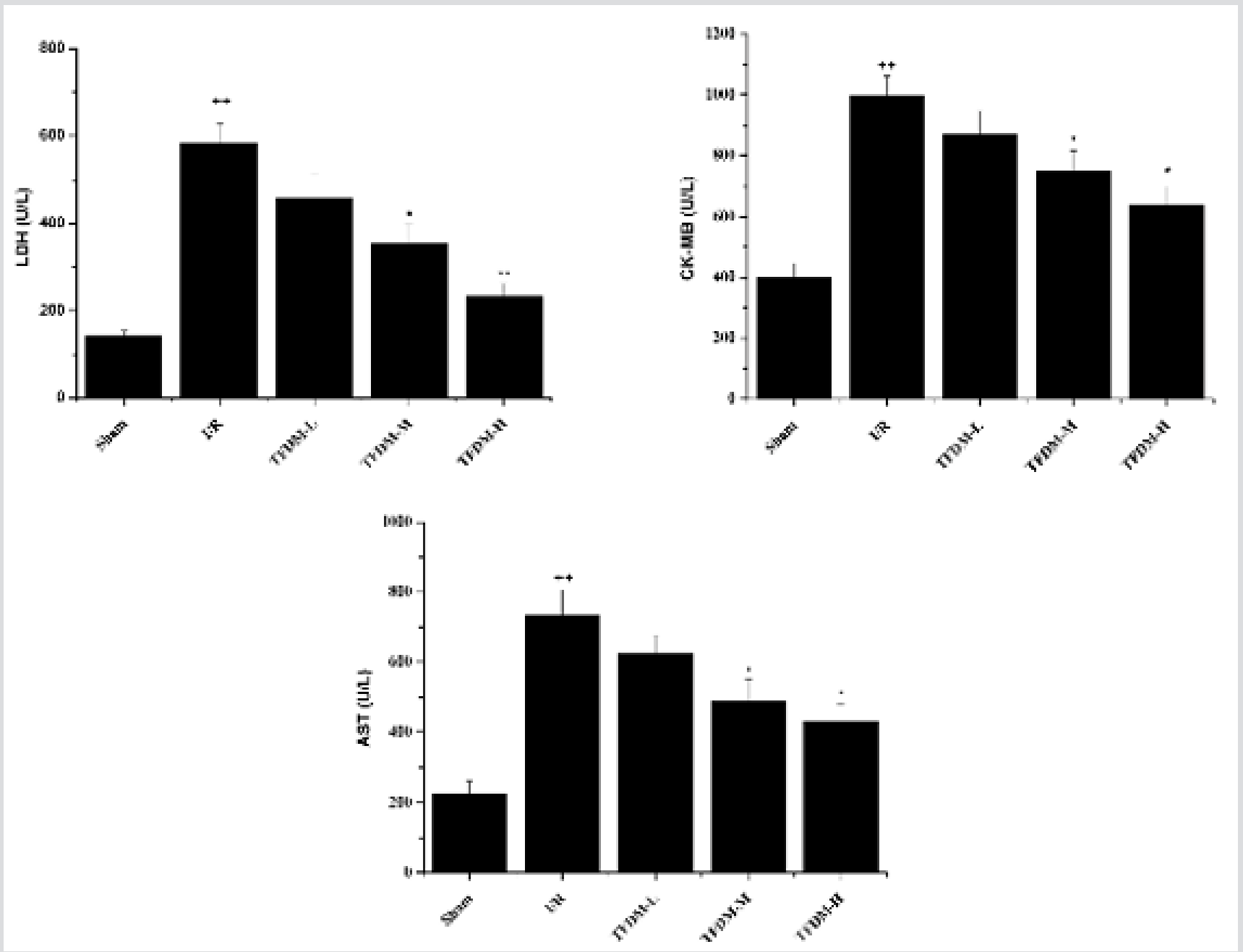

Figure 5: Effects of TFDM on CK-MB, LDH and AST in serum. Compared with Sham group, $++\mathrm{P}<0.01$; Compared with I/R group, ${ }^{*} \mathrm{P}<0.05, * * \mathrm{P}<0.01$.

\section{Effects of TFDM Treatment on Apoptosis both in vivo and in vitro}

As indicated by the red arrows in Figure 6a, the apoptotic myocardial nucleus of TUNEL-positive was stained in brown. Instead, the nucleus of normal cardiac myocytes had blue-staining. The cells apoptotic index was showed in Figure 6b. Compared with Sham group, the apoptosis rate in I/R injury group obviously increased. However, the percentage of apoptotic cells was decreased evidently in the TFDM treated group at either 30 or $60 \mathrm{mg} / \mathrm{kg}$ versus to I/R group. These experimental data clearly suggested that TFDM prevented cardiomyocyte apoptosis induced by I/R. Moreover, compared with the TFDM-H group, the high dosage of TFDM treatment combined with LY294002 (PI3K inhibitor) and PD98059 (Erk1/2 inhibitor) aggravated apoptosis induced by I/R. On the contrary, apoptosis of cardiac myocyte was alleviated by SB203580 (P38 MAPK inhibitor). Taken above results into consideration, PI3K/Akt, Erk 1/2 and P38 MAPK signal pathway may be involved in cardioprotection of TFDM in the process of resisting MIRI. 


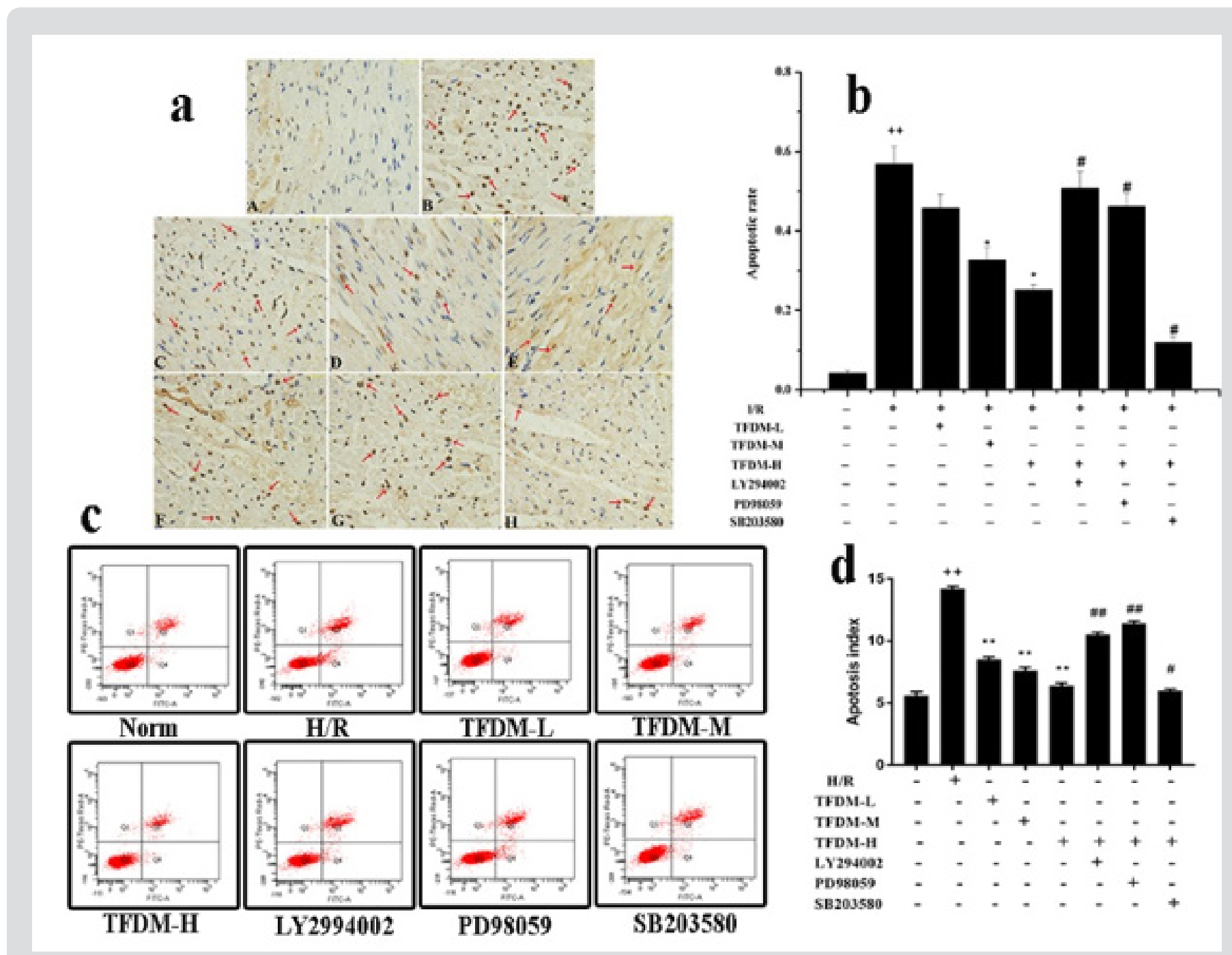

Figure 6: Effects of TFDM treatment on apoptosis both in vivo and in vitro. (a) Effects of TFDM on apoptosis detected by TUNEL in rats. A represented Sham groups. B represented I/ R group. C represented TFDM-L group. D represented TFDM-M group. E represented TFDM-H group. F represented TFDM-H combined with LY294002 group. G represented TFDM-H combined with PD98059 group. H represented TFDM-H combined with SB203580 group. (b) Graph of apoptosis rate in vivo. (c) Effects of TFDM on apoptosis detected by Flow cytometry in cells. (d) Graph of apoptosis rate in vitro. Compared with the Sham or Norm group, $++\mathrm{P}<0.01$; Compared with I/ $\mathrm{R}$ or H/R group, ${ }^{*} \mathrm{P}<0.05,{ }^{* *} \mathrm{P}<0.01$. Compared with the TFDM-H group, $\# \mathrm{P}<0.05, \# \# \mathrm{P}<0.01$.

\section{Effects of TFDM Treatment on Apoptosis-Related Pro- teins in I/R Cardiac Tissue}

To further investigate the effect of TFDM on apoptosis, we analyzed the expression of Bcl-2, Bax, cleaved-capase-3 and cytochrome $\mathrm{c}$ in cytoplasm. I/R injury caused the activation of apoptotic pathway, in which cleaved-capase-3 and cytochrome c were up-regulated while the ratio of $\mathrm{Bcl}-2 / \mathrm{Bax}$ was down-regulated. However, pretreatment with TFDM significantly increased Bcl-2 and decreased Bax, cleaved-capase- 3 and cytochrome c expressions versus to the I/R group, especially in TFDM-M and TFDM-H groups (Figure 7).

\section{Effects of TFDM on the Expressions of PI3K/Akt, Erk 1/2 and P38 MAPK Pathway Related Proteins}

In order to further explore potential protection mechanism, the expressions of Akt, Erk1/2 and p38 MAPK pathway-related proteins were also examined in myocardium and H9c2 cell. As shown in Figure 8 , the levels of p-Akt, p-Erk, p-Gsk-3 $\beta$ were decreased in MIRI rats versus to Sham group, taking the expressions of Akt, Erk and Gsk-3 $\beta$ as inner controls respectively, while different extents of upregulation of them were found in TFDM groups, which was tightly related to the dosage of administration. Meanwhile, pretreatment with TFDM significantly decreased the phosphorylation of p38 and inhibit the activation of p38 MAPK pathway (Figure 8). 


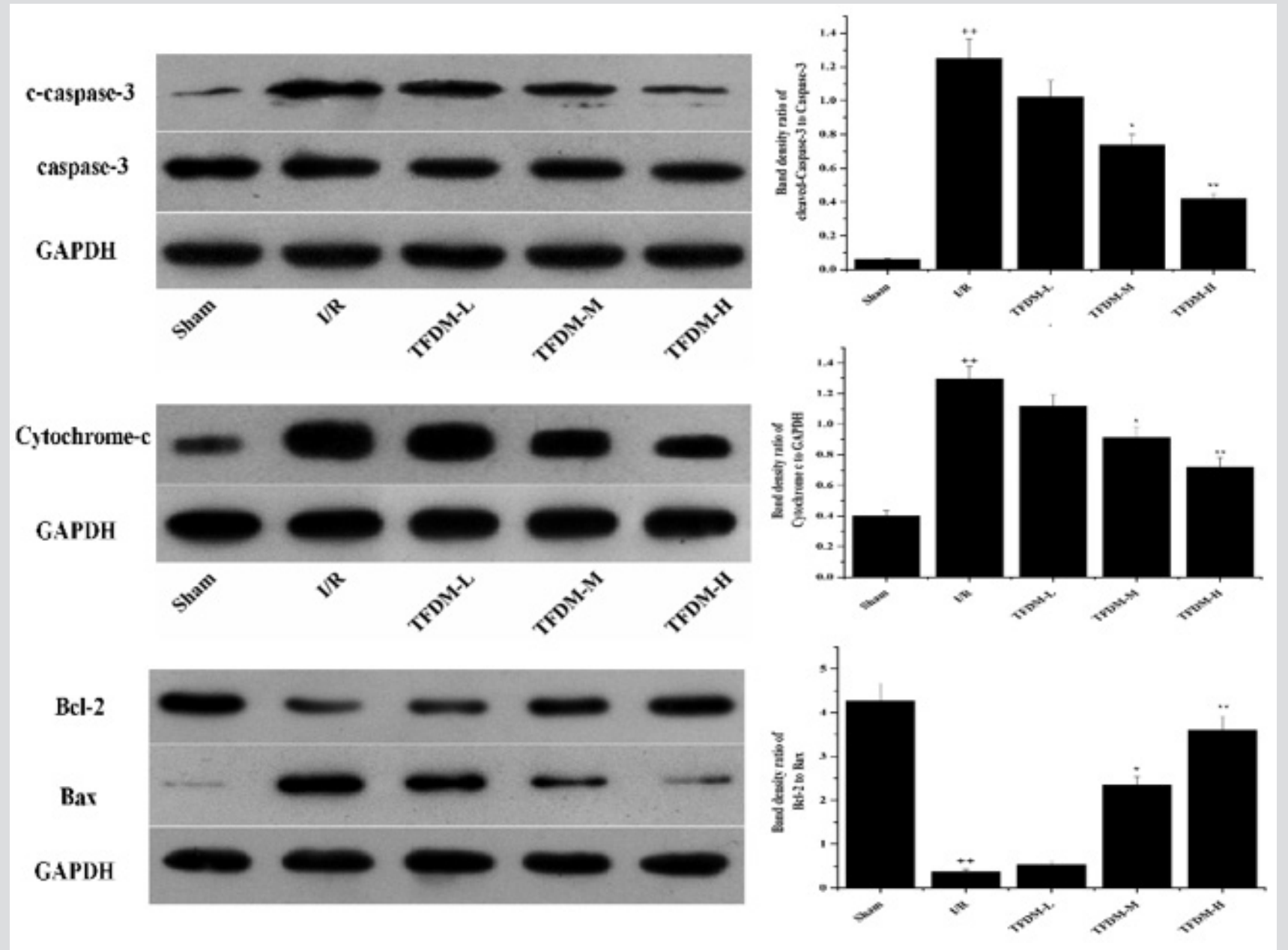

Figure 7: Effect of TFDM treatment on apoptosis-related proteins in I/R cardiac tissue. Compared with the Sham group, $++\mathrm{P}<0.01$; Compared with the I/R group, ${ }^{*} \mathrm{P}<0.05,{ }^{* *} \mathrm{P}<0.01$. 


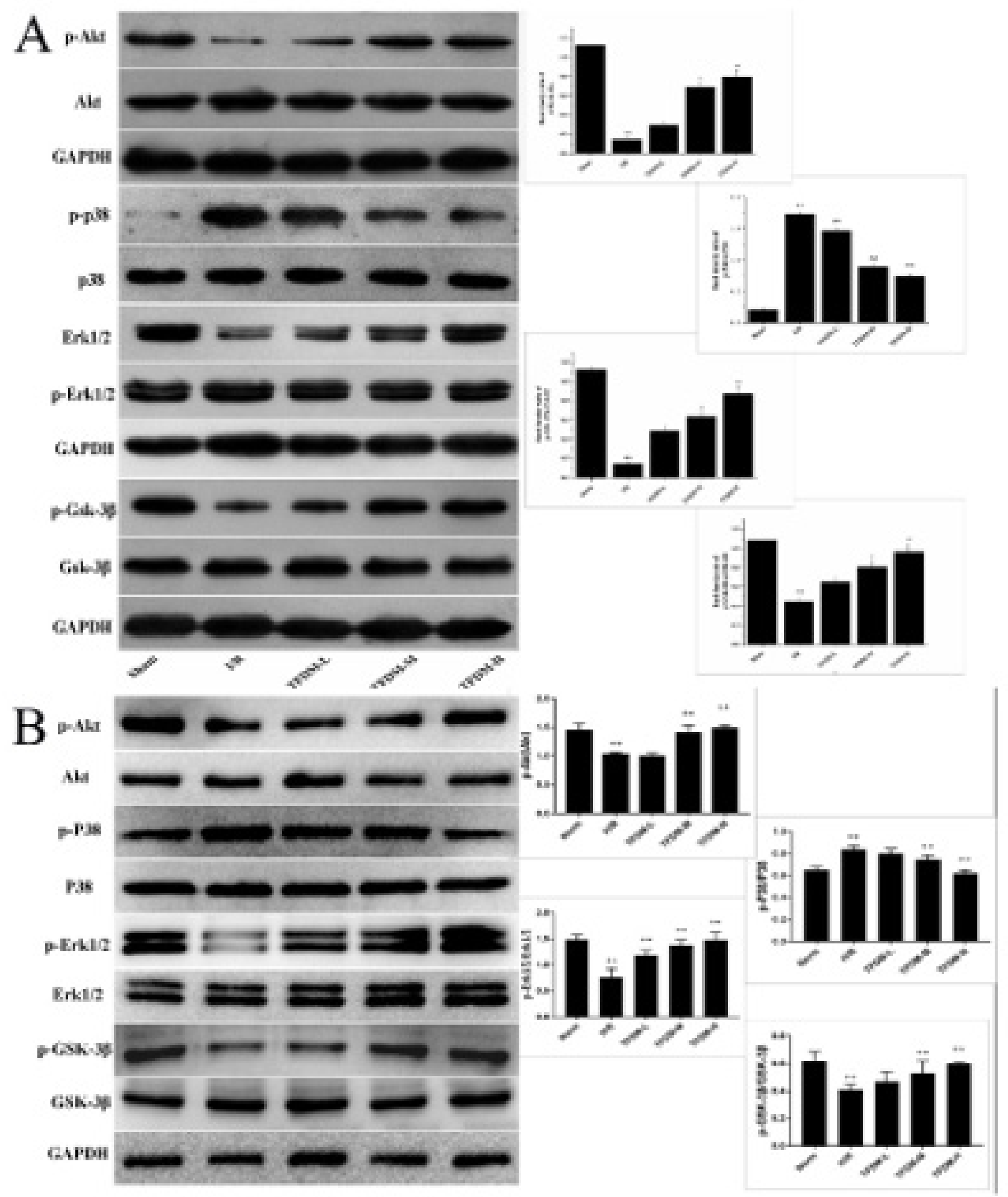

Figure 8: Effect of TFDM treatment on the expressions of RISK and P38 MAPK pathway related proteins. A was the expressions of proteins in vivo. $\mathrm{B}$ was the expressions of proteins in vitro. Compared with the Sham or Norm group, $++\mathrm{P}<0.01$; Compared with the I/ $\mathrm{R}$ or $\mathrm{H} / \mathrm{R}$ group, ${ }^{*} \mathrm{P}<0.05,{ }^{* *} \mathrm{P}<0.01$. 


\section{Discussion}

As mentioned in results, TFDM has been demonstrated to have an obvious cardioprotective against MIRI in vivo and vitro study. The establishment of MIRI model was confirmed by measurement of infract size, morphological and histological examination for myocardium, and change of myocardial enzymes. Firstly, we found that rats with MIRI exhibited an extensive infarct size, which led to severe myocardial structural disorder and massive leakage of LDH, CK-MB and AST. Nevertheless, pretreatment with TFDM could attenuate the myocardial injury while decrease the enzymes of serum in dose-dependent manners and increased the H9c2 cell viability. On the one hand, the apoptosis rates in rats and $\mathrm{H} 9 \mathrm{c} 2$ cell were decreased by TFDM administration. On the other hand, related apoptosis proteins were detected, and proofs conclusively displayed that TFDM down-regulated the expression of cytochrome $c$ and restrained the activation of caspase- 3 . In addition, the ratio of Bcl-2/Bax was raised after TFDM administration as well, which indirectly reflected the regulation of TFDM on apoptosis.

We also evaluated the effects of Akt, Erk1/2 and p38 related inhibitors and various dosage of TFDM on cardiomyocyte apoptosis induced by $I / R$ and $H / R$, the anti-apoptotic effect of TFDM was revered by PI3K and Erk1/2 inhibitors, whereas the p38 MAPK inhibitor conversely eased its effect. Based on the analysis of related proteins, the experiment further confirmed that the modulation of PI3K, Erk1/2 and p38 MAPK signaling pathway on apoptosis might be associated with cardioprotective of TFDM. CK-MB, LDH, and AST are enzymes presenting in the cytoplasm of cardiomyocytes, which are rapidly released into the blood stream when the myocyte suffers from necrotic damage or destruction of cellular membrane [20]. Therefore, these enzymes are accepted as serum biomarkers that reflect myocardial damage [21,22]. Our research showed that TFDM obviously decreased the leakage of LDH, CK-MB and AST.

The studies of Baliga et al. [24] indicated that myocardial ischemia/reperfusion (I/R) injury is prone to resulting in cardiomyocyte apoptosis [23,24]. Mitochondria-mediated cardiomyocyte apoptosis is triggered by excessive ROS and $\mathrm{Ca}^{2+}$ in early reperfusion, which causes the mitochondrial permeability transition pore (mPTP) opened [25-27]. Consistent opening of the mPTP results in mitochondrial matrix swelling, ultimately leads to rupture of the outer membrane and releases of cytochrome $\mathrm{c}$ to the cytosol, where it activates the caspase- 3 into cleaved-caspase-3, which is regarded as an important executor of programmed cell death [28,29]. Bcl-2 family members including pro-apoptotic (Bax) and anti-apoptotic (Bcl-2) proteins regulates apoptosis by interacting with MPTP to modulate the mitochondrial membrane permeability. Therefore, the balance of the Bcl-2 and Bax protein is a key factor in determining whether cells undergo apoptosis or survive [7,30,31]. Our experimental results indicated that TFDM elevated the ratio of Bcl2/Bax and inhibited the apoptosis, which was consistent with a significant decrease in the number of apoptotic cells in I/R rats and H9c2 cell treated with TFDM.
The RISK pathway including PI3K/Akt and Erk1/2 has been demonstrated to be a crucial signaling pathway for survival of myocardium in the period of reperfusion, the activation of which exerts powerful protection against I/R injury via limiting apoptotic cell death events in response to injurious stimuli $[32,33]$. The Gsk$3 \beta$ is well known as a central downstream effector of the RISK pathway, which is phosphorylated by Akt and Erk1/2 [34,35]. With the activation of PI3K/Akt and Erk1/2 signaling pathway, Akt as well as Erk1/2 usually promotes cardiomyocyte survival by phosphorylation of Gsk-3 $\beta$ at Ser9, which leads to inhibit the mPTP opening and then inactivate apoptotic pathway depended on mediation of mitochondria [36,37]. Our data showed that TFDM increased phosphorylation of Akt, Erk1/2 and Gsk-3 $\beta$. These results confirmed that GSK-3 $\beta$ is an important downstream protein of PI3K/Akt and Erk1/2 pathway and suggested the vital role of the RISK/ GSK3 $\beta$ pathway in TFDM-induced protection.

Besides Erk1/2/GSK-3 $\beta$ pathway, P38 MAPK also plays distinct and complementary roles in MIRI. The difference between Erk1/2 and P38 MAPK is that Erk1/2 confers to powerful protection for cardiomyocyte, but P38 MAPK acts as an adverse effect, which increases the adhesion molecules and production of cytokines $[5,38]$. In this study, pretreatment with TFDM markedly reduced the phospho-P38 level, indicating that TFDM could inhibit the P38 MAPK pathway in MIRI. To sum up, our experiments provided credible evidence to support protective effects of TFDM on apoptosis in MIRI. TFDM exerted anti-apoptosis effect against MIRI in vivo and vitro, which was related with the activation of RISK/ GSK-3 $\beta$ pathway and suppression of p38 MAPK signal pathway.

\section{Acknowledgment}

This work was supported by Grants from the National Natural Science Foundation of China (Nos.81660704 and 81860747).

\section{Conflicts of Interest}

The authors declare no conflict of interest.

\section{References}

1. Thygesen K, Alpert JS, White HD, Joint ESCAAHAWHFTFftRoMI (2007): Universal definition of myocardial infarction. European heart journal 28(20): 2525-2538.

2. Wu YH, Liu W, Zhang L, Liu XY, Wang Y, etal. (2018) Effects of microRNA-24 targeting C-myc on apoptosis, proliferation, and cytokine expressions in chondrocytes of rats with osteoarthritis via MAPK signaling pathway. Journal of cellular biochemistry 119(10): 7944-7958.

3. Moens AL, Claeys MJ, Timmermans JP, Vrints CJ (2005) Myocardial ischemia/reperfusion-injury, a clinical view on a complex pathophysiological process. International journal of cardiology 100(2): 179-190.

4. Hausenloy DJ, Yellon DM (2013) Myocardial ischemia-reperfusion injury: a neglected therapeutic target. The Journal of clinical investigation 123(1): 92-100.

5. Jeong CW, Yoo KY, Lee SH, Jeong HJ, Lee CS, et al. (2012) Curcumin protects against regional myocardial ischemia/reperfusion injury through activation of RISK/GSK-3beta and inhibition of p38 MAPK and JNK. Journal of cardiovascular pharmacology and therapeutics 17(4): 387-394. 
6. Chen Y, Ba L, Huang W, Liu Y, Pan H, et al. (2017) Role of carvacrol in cardioprotection against myocardial ischemia/reperfusion injury in rats through activation of MAPK/ERK and Akt/eNOS signaling pathways. European journal of pharmacology 796: 90-100.

7. Cheng J, Zhou ZW, Sheng HP, He LJ, Fan XW, et al. (2015) An evidencebased update on the pharmacological activities and possible molecular targets of Lycium barbarum polysaccharides. Drug design, development and therapy 9: 33-78.

8. Xing J, Peng K, Cao W, Lian X, Wang Q et al. (2013) Effects of total flavonoids from Dracocephalum moldavica on the proliferation migration, and adhesion molecule expression of rat vascular smooth muscle cells induced by TNF-alpha. Pharmaceutical biology 51(1): 74 83.

9. Martinez Vazquez M, Estrada Reyes R, Martinez Laurrabaquio A, Lopez Rubalcava C, Heinze G (2012) Neuropharmacological study of Dracocephalum moldavica L. (Lamiaceae) in mice: sedative effect and chemical analysis of an aqueous extract. Journal of ethnopharmacology 141(3): 908-917.

10. Lv D, Cheng X, Tang L, Jiang M (2017) The cardioprotective effect of total flavonoids on myocardial ischemia/reperfusion in rats. Biomedicine \& pharmacotherapy 88: 277-284.

11. Li Q Liu Y, Han L, Liu J, Liu W, et al. (2016) Chemical constituents and quality control of two Dracocephalum species based on highperformance liquid chromatographic fingerprints coupled with tandem mass spectrometry and chemometrics. Journal of separation science 39(21): 4071-4085.

12. Jiang J, Yuan X, Wang T, Chen H, Zhao H, et al. (2014) Antioxidative and cardioprotective effects of total flavonoids extracted from Dracocephalum moldavica L. against acute ischemia/reperfusion-induced myocardial injury in isolated rat heart. Cardiovascular toxicology 14(1): 74-82.

13. Xinmei F, Wenjiang C, Jianguo X (2013) Protective effect of tota flavones from Dracocephalum moldavica against myocardial ischemiareperfusion injury in rats. Chin Trad Patent Med 35: 1625-1629.

14. Yu N, He C, Awuti G, Zeng C, Xing J, et al. (2015) Simultaneous Determination of Six Active Compounds in Yixin Badiranjibuya Granules, a Traditional Chinese Medicine, by RP-HPLC-UV Method. Journal of analytical methods in chemistry 2015: 974039.

15. Yuan Y, Cao W, Hong Y, Guo X, Wang Y, et al. (2017) Tilianin pretreatment prevents myocardial ischemia-reperfusion injury via preservation of mitochondrial function in rat heart. Phytomedicine 34: 106-114.

16. He Y, Li C, Ma Q Chen S (2018) Esculetin inhibits oxidative stress and apoptosis in $\mathrm{H} 9 \mathrm{c} 2$ cardiomyocytes following hypoxia/reoxygenation injury. Biochemical and biophysical research communications 501(1): 139-144.

17. Wang Y, Yuan Y, Wang X, Wang Y, Cheng J, et al. (2017) Tilianin PostConditioning Attenuates Myocardial Ischemia/Reperfusion Injury via Mitochondrial Protection and Inhibition of Apoptosis. Medical science monitor 23: 4490-4499.

18. Wang J, Yang H, Hu X, Fu W, Xie J, et al. (2013) Dobutamine-mediated heme oxygenase-1 induction via P13K and p38 MAPK inhibits high mobility group box 1 protein release and attenuates rat myocardial ischemia/reperfusion injury in vivo. The Journal of surgical research 183(2): 509-516.

19. Tao J, Zhu W, Li Y, Xin P, Li J, et al. (2011) Apelin-13 protects the heart against ischemia-reperfusion injury through inhibition of ER-dependent apoptotic pathways in a time-dependent fashion. American journal of physiology. Heart and circulatory physiology 301(4): H1471-H1486.

20. Prasad EM, Mopuri R, Islam MS, Kodidhela LD (2017) Cardioprotective effect of Vitex negundo on isoproterenol-induced myocardial necrosis in wistar rats: A dual approach study. Biomedicine \& pharmacotherapy 85: 601-610.

21. Yu D, Li M, Tian Y, Liu J, Shang J (2015) Luteolin inhibits ROS-activated MAPK pathway in myocardial ischemia/reperfusion injury. Life sciences 122: $15-25$
22. Ge G, Zhang Q, Ma J, Qiao Z, Huang J, et al. (2014) Protective effect of Salvia miltiorrhiza aqueous extract on myocardium oxidative injury in ischemic-reperfusion rats. Gene 546(1): 97-103.

23. Eltzschig HK, Eckle T (2011) Ischemia and reperfusion--from mechanism to translation. Nature medicine 17(11): 1391-1401.

24. Baliga RR (2001) Apoptosis in myocardial ischemia, infarction, and altered myocardial states. Cardiology clinics 19(1): 91-112.

25. Miura T, Tanno M, Sato T (2010) Mitochondrial kinase signalling pathways in myocardial protection from ischaemia/reperfusioninduced necrosis. Cardiovascular research 88(1): 7-15

26. Sanada S, Komuro I, Kitakaze M (2011) Pathophysiology of myocardial reperfusion injury: preconditioning, postconditioning, and translational aspects of protective measures. American journal of physiology. Heart and circulatory physiology 301(5): H1723-1741.

27. Tawa P, Hell K, Giroux A, Grimm E, Han Y, et al. (2004) Catalytic activity of caspase- 3 is required for its degradation: stabilization of the active complex by synthetic inhibitors. Cell death and differentiation 11(4): 439-447.

28. Hausenloy DJ, Yellon DM (2007) The evolving story of "conditioning" to protect against acute myocardial ischaemia-reperfusion injury. Heart 93(6): 649-651.

29. Ibanez B, Heusch G, Ovize M, Van de Werf F (2015) Evolving therapies for myocardial ischemia/reperfusion injury. Journal of the American College of Cardiology 65(14): 1454-1471.

30. Kim HS, Kim SY, Kwak YL, Hwang KC, Shim YH (2012) Hyperglycemia attenuates myocardial preconditioning of remifentanil. The Journal of surgical research 174(2): 231-237.

31. Zhao ZQ Nakamura M, Wang NP, Wilcox JN, Shearer S, et al. (2000) Reperfusion induces myocardial apoptotic cell death. Cardiovascular research 45(3): 651-660

32. Hausenloy DJ, Yellon DM (2004) New directions for protecting the heart against ischaemia-reperfusion injury: targeting the Reperfusion Injury Salvage Kinase (RISK)-pathway. Cardiovascular research 61(3): 448460

33. Yin Y, Guan Y, Duan J, Wei G, Zhu Y, et al. (2013) Cardioprotective effect of Danshensu against myocardial ischemia/reperfusion injury and inhibits apoptosis of H9c2 cardiomyocytes via Akt and ERK1/2 phosphorylation. European journal of pharmacology 699(1-3): 219-226

34. Hausenloy DJ, Tsang A, Yellon DM (2005) The reperfusion injury salvage kinase pathway: a common target for both ischemic preconditioning and postconditioning. Trends in cardiovascular medicine 15(2): 69-75.

35. Penna C, Perrelli MG, Pagliaro P (2013) Mitochondrial pathways, permeability transition pore, and redox signaling in cardioprotection: therapeutic implications. Antioxidants \& redox signaling 18(5): 556599.

36. Park SS, Zhao H, Mueller RA, Xu Z (2006) Bradykinin prevents reperfusion injury by targeting mitochondrial permeability transition pore through glycogen synthase kinase 3 beta. Journal of molecular and cellular cardiology 40: 708-716.

37. Song JQ, Teng X, Cai Y, Tang CS, Qi YF (2009) Activation of Akt/GSK-3beta signaling pathway is involved in intermedin(1-53) protection against myocardial apoptosis induced by ischemia/reperfusion. Apoptosis 14(11): 1299-1307.

38. Jeong JJ, Ha YM, Jin YC, Lee EJ, Kim JS, et al. (2009) Rutin from Lonicera japonica inhibits myocardial ischemia/reperfusion-induced apoptosis in vivo and protects $\mathrm{H} 9 \mathrm{c} 2$ cells against hydrogen peroxide-mediated injury via ERK1/2 and PI3K/Akt signals in vitro. Food and chemical toxicology 47(7): 1569-1576. 
ISSN: 2574-1241

DOI: 10.26717/BJSTR.2019.20.003444

Xinchun Wang, Xinchun Wang. Biomed J Sci \& Tech Res

(C) (P) This work is licensed under Creative

Submission Link: https://biomedres.us/submit-manuscript.php

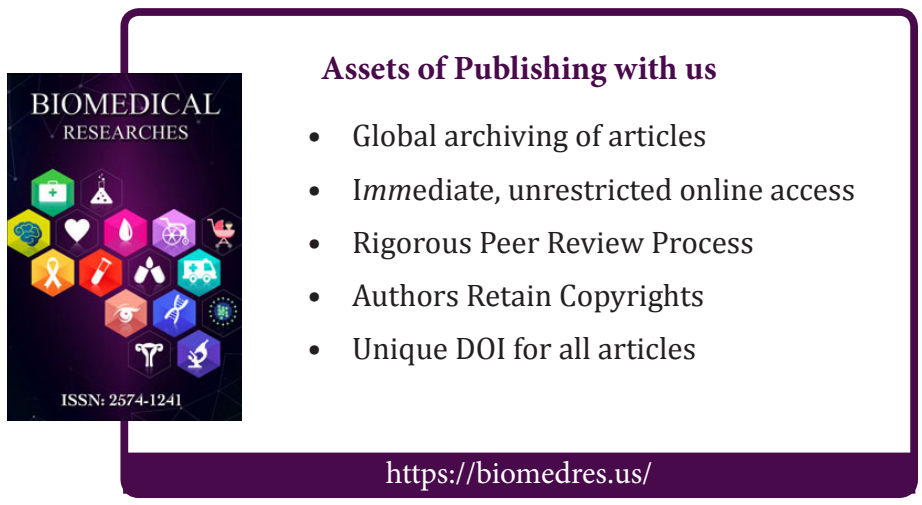

